\title{
ESTIMACIÓN DE PARÁMETROS DE UN MODELO DE MANIOBRA NO LINEAL DE UN VEHÍCULO SUBACUÁTICO TELEOPERADO MEDIANTE ENSAYOS EN CANAL
}

\section{Resumen}

En este trabajo, se aplican técnicas de inferencia indirecta para la estimación de parámetros de un modelo de maniobra no lineal de un vehículo subacuático teleoperado (ROV). Para ello, se dispone de datos de diferentes ensayos, que se llevaron a cabo con el citado ROV en las instalaciones del Canal de Experiencias Hidrodinámicas del Pardo CEHIPAR, Madrid. El modelo obtenido se valida mediante métodos gráficos y estadísticos y las propiedades estadísticas de los parámetros estimados se evalúan mediante un estudio de Monte Carlo.
\end{abstract}

Elías Revestido Herrero

E. T. S. de Náutica, c $\backslash$ Gamazo 1, 39004, Santander, revestidoe@unican.es

José Ramón Llata García

E.T.S. de Ingenieros Industriales y de Telecomunicación, Av. de los Castros s/n- 39005 Santander, llataj@unican.es

Francisco Jesús Velasco González

E. T. S. de Náutica, c \Gamazo 1, 39004, Santander, velascof@unican.es

José Joaquín Sainz Gutiérrez

E.T.S. de Ingenieros Industriales y de Telecomunicación, Av. de los Castros s/n- 39005 Santander, josejoaquin.sainz@unican.es

Patricia Diaz Torrijos

Instituto Nacional de Técnica Aeroespacial (INTA), Subdirección General de Sistemas Navales, C/ Adelina Patti S/N, 28048 El Pardo-Madrid, diaztp@inta.es

Palabras clave: modelo de maniobra no lineal; inferencia indirecta; ROV; mínimos cuadrados ordinarios.

\section{INTRODUCCIÓN}

La aplicación de vehículos no tripulados en el ámbito naval se conoce ampliamente en el ámbito científico, uno de los sectores que están impulsando esta tecnología con más fuerza en los últimos años es el militar y de seguridad [9]. La incorporación de vehículos no tripulados en Defensa, han contribuido al estado del arte de los sistemas no tripulados [8] para misiones peligrosas o de alto riesgo, tales como el seguimiento, la detección y neutralización de minas. Hoy en día, los autonomous underwater vehicles-unmanned underwater vehicles (AUV-
UUVs) son de suma importancia, tanto para aplicaciones y procedimientos para la exploración submarina en aplicaciones de defensa y civiles.

Para este tipo de aplicaciones con vehículos no tripulados, en primer lugar es muy habitual, con objeto de desarrollar posteriormente un sistema de control adecuado, la identificación de modelos de maniobra no-lineales de seis grados de libertad (6GDL) obtenidos a partir de ensayos. En la literatura se encuentran diferentes tipos de técnicas para la obtención de estos modelos, ver [2][10] y sus referencias. Sin embargo, existen técnicas que todavía apenas se han aplicado a este cometido como son las técnicas de inferencia indirecta. Estas técnicas se han utilizado ampliamente para el modelado de series temporales en el área de econometría [4].

Posteriormente, las técnicas de inferencia indirecta se adaptaron para incluir sistemas con entradas exógenas y para aplicarlas al problema de identificación de sistemas [12]. Este último enfoque es el que aplica en este trabajo, para la estimación de parámetros de un modelo de maniobra no lineal de un vehículo subacuático teleoperado. En la literatura se encuentran trabajos en los que se aplican estas técnicas de inferencia indirecta a un modelo no lineal de tanques [5] y también a un modelo en el dominio de la frecuencia de una maquina síncrona [1]. Por otra parte, se encuentra un trabajo en la literatura que aplica tecnicas de inferecia indirecta a un AUV [13]. Además este método proporciona mejores resultados en lo que se refiere a la exactitud y a la eficiencia de los parámetros estimados, con respecto a otros 
métodos existentes en la literatura como pueda ser el método de variables instrumentales [14].

En el presente trabajo se aplican técnicas de inferencia indirecta a la estimación de parámetros de un modelo de maniobra no lineal correspondiente a un vehículo teleoperado. Se proponen dos casos de estudio uno con datos simulados para verificar las propiedades estadísticas del método aplicado y otro con datos reales adquiridos en las instalaciones del Canal de Experiencias Hidrodinámicas del Pardo CEHIPAR, donde se valida el modelo con métodos gráficos y estadísticos.

\section{MODELO DE MANIOBRA NO LINEAL}

El modelo de maniobra no lineal de 6 GDL se puede expresar de la siguiente forma [3]:

$M v v^{\prime}+C(v) v+D(v) v+g(\eta)=\tau$

$y=\eta+v, \eta^{\prime}=J(\eta) v$

donde $\eta=[x, y, z, \phi, \theta, \psi]^{T}$ es el vector de posición $\mathrm{y}$ ángulos de Euler, $v^{\prime}=[u, v, w, p, q, r]^{T}$ son las velocidades lineales y angulares, $\tau=[X, Y, Z, K, M, N]^{T}$ son las fuerzas y momentos $v$ es el ruido en la medición. $M$ es la matriz de masa adicional, $C(v) v$ es el término de Coriolis, $g(\eta)$ es la matriz de restauración, $J(\eta)$ es la matriz de rotación y $D(v) v$ representa el amortiguamiento hidrodinámico, que es una combinación de términos lineales y no lineales. Todos los parámetros de las matrices de la ecuación (1) considerando las simetrías del vehículo objeto de estudio de este articulo (ver figura 1) se pueden encontrar en Fossen [3].

\section{ENSAYOS EN CANAL}

En esta sección se enumeran las pruebas que se realizaron en el Canal de Experiencias Hidrodinámicas de El Pardo (CEHIPAR) para la estimación de parámetros del modelo matemático que se ha definido anteriormente. Para ello, se ha utilizado un ROV comercial, cuyas dimensiones son de $1 \mathrm{~m} \times 0,6 \mathrm{~m}, 0,62 \mathrm{~m}$. En la figura 1 se muestra el montaje del vehículo en el canal de aguas tranquilas del CEHIPAR. Los ensayos consistieron en hacer distintos movimientos controlados del ROV mediante dos actuadores lineales controlados por ordenador y montados en el carro de remolque.

Las pruebas realizadas fueron las siguientes:

- Resistencia y aceleración en longitudinal a 3 velocidades.

- Autopropulsión para cada hélice (velocidad cero y navegación nominal).

- Pitch estático a 3 ángulos (velocidad de navegación nominal).
- Heave dinámico a una frecuencia y tres amplitudes (velocidad cero y navegación nominal).

- Pitch dinámico a una frecuencia y tres amplitudes (velocidad cero y navegación nominal).

- Yaw estático a 3 ángulos (velocidad de navegación nominal).

- Sway dinámico a una frecuencia y tres amplitudes (velocidad cero y navegación nominal).

- Yaw dinámico a una frecuencia y tres amplitudes (velocidad cero y navegación nominal).

(para más detalles sobre este tipo de ensayos ver Revestido [11] y Phillips [7]).

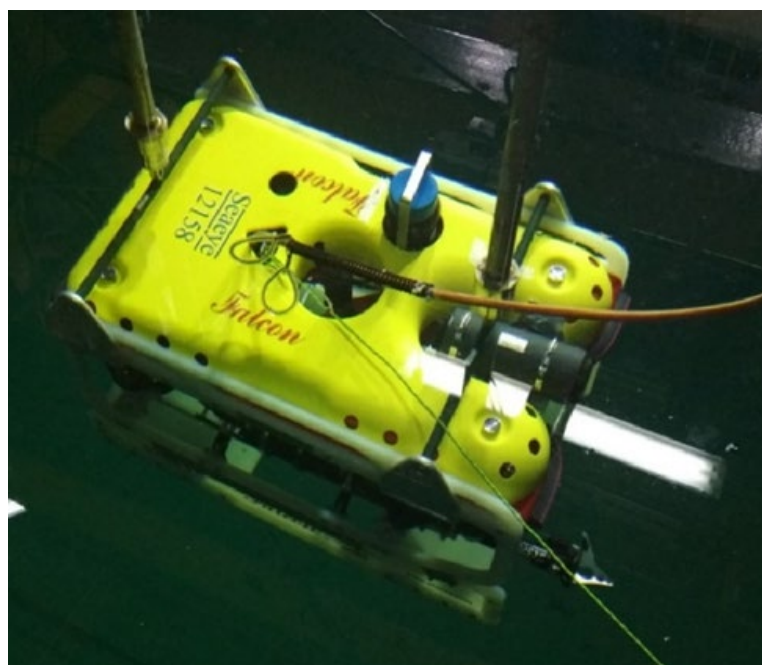

Figura 1: Montaje del ROV en las instalaciones del CEHIPAR

En los distintos ensayos se midieron las aceleraciones transversal y vertical con 2 acelerómetros, uno en cada extremo final de cada cilindro. También se midieron directamente las fuerzas que actuaban en los 2 puntos de unión del ROV con las barras de prolongación de los cilindros, ver Figura 1.

El centro de gravedad y las inercias del ROV se obtuvieron de forma experimental mediante oscilaciones forzadas. Los valores obtenidos se reflejan en la Tabla 1 .

\begin{tabular}{|c|c|c|}
\hline Magnitud & Unidad & Valor \\
\hline Peso & $\mathrm{Kg}$ & 80.750 \\
$\mathbf{X g}$ & $\mathrm{m}$ & 0.490 \\
$\mathbf{Y g}$ & $\mathrm{m}$ & 0.288 \\
$\mathbf{Z g}$ & $\mathrm{m}$ & 0.400 \\
Itransversal & $\mathrm{kg}^{*} \mathrm{~m} 2$ & 5.453 \\
Ilongitudinal & $\mathrm{kg}^{*} \mathrm{~m} 2$ & 10.499 \\
\hline
\end{tabular}

Tabla 1 Distribución de masas del ROV. 
Las inercias están referidas al centro de gravedad estimado. La inercia en guiñada se ha supuesto igual a la longitudinal.

\section{ESTIMACIÓN DE PARÁMETROS}

El método de inferencia indirecta utiliza un modelo "mal especificado" cuyos parámetros se pueden estimar mediante datos del sistema verdadero. El método también utiliza un modelo parametrizado cuya estructura es conocida y corresponde a la estructura real del sistema (en este caso el modelo de maniobra ecuación (1)), este modelo se emplea para obtener datos simulados con el modelo real. El objetivo del método es obtener los parámetros del modelo real que minimicen la distancia entre los parámetros obtenidos del modelo mal especificado con datos del sistema verdadero y los parámetros obtenidos con el modelo mal especificado con datos simulados. En la Figura 2 se muestra un diagrama de bloques del método de inferencia indirecta.

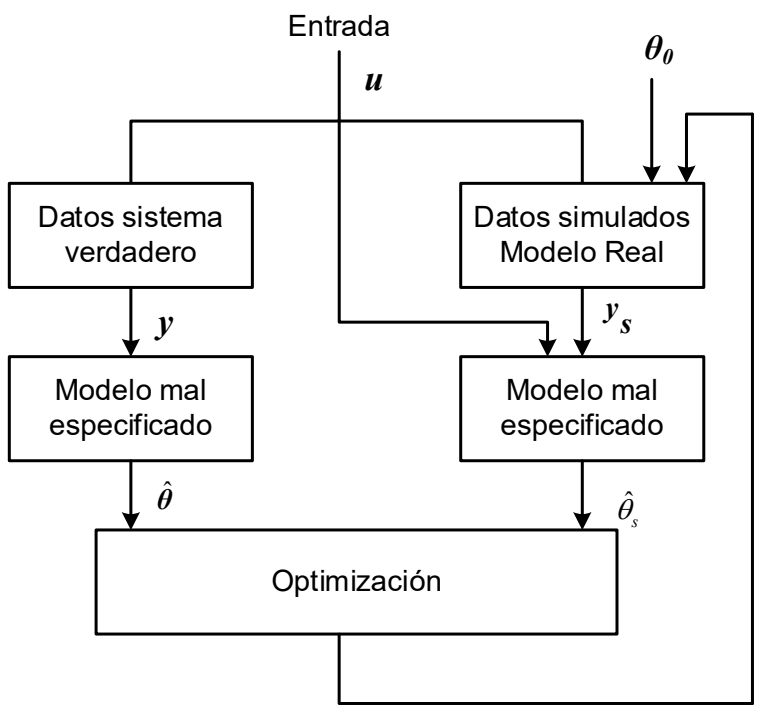

Figura 2: Diagrama de bloques del método de inferencia indirecta.

Para la estimación de los parámetros del modelo mal especificado estimado con datos del sistema verdadero y el modelo mal especificado estimado con dato simulados del modelo real (ver figura 2), se aplica el método de mínimos cuadrados ordinarios.

Todos los parámetros que se han estimado, se refirieren a un sistema de coordenadas en el centro de gravedad del vehículo, ver Tabla 1. Para la estimación de los parámetros se ha planteado, el modelo clásico de regresión lineal, que se puede escribir con la siguiente notación [6]:

$y=\varphi \theta+w$ $y=\left[\begin{array}{ll}y_{1} & y_{2} \ldots y_{N}\end{array}\right]=N \times 1$ vector de mediciones de salida.

$\theta=\left[\begin{array}{ll}\theta_{0} & \theta_{1} \ldots \theta_{n}\end{array}\right]=n_{p} \times 1$ vector de parámetros desconocidos, $n_{r}=n_{p}+1 \varphi=\left[\begin{array}{llll}1 & \varphi_{1} & \varphi_{2} \ldots & \varphi_{n}\end{array}\right]=N \times n_{r}$ matriz de regresores.

$w=\left[\begin{array}{ll}w_{1} & w_{2} \ldots w_{N}\end{array}\right]=N \times 1$ vector de errores en la medición.

$N=$ Número de muestras.

$n_{p}=$ Número de parámetros

donde $\theta_{0}$ es el parámetro de sesgo, que se corresponde con el regresor de unos incluido en $\varphi$. Es conveniente introducir en el modelo este parámetro, para estimar el posible sesgo que puedan tener los datos, incluso en el caso de que se obtengan valores de $\theta_{0}$ próximos a cero.

\section{RESULTADOS}

En este artículo se proponen dos casos de estudio para la estimación de la fuerza de avance de la ecuación (1): uno de ellos con datos simulados y el otro con datos adquiridos en las instalaciones del CEHIPAR. En el primero de ellos se verifican las propiedades estadísticas del estimador basado en inferencia indirecta mediante un estudio de Monte Carlo de 100 realizaciones.

El modelo mal especificado se conforma eliminando el término no lineal de la ecuación de la fuerza de avance. Los datos del sistema verdadero se han generado en base a un modelo estimado con datos del CEHIPAR, el cual se ha simulado y se le añadido niveles de ruido de la instrumentación estándar a bordo de un ROV. En la tabla 1 se muestran los resultados de este estudio para la fuerza de avance, donde se observa como el método de inferencia converge a los parámetros verdaderos con valores de sesgo inferiores al $1 \%$. Además, los valores bajos de desviación estándar de cada uno de los parámetros indican que el método aplicado es eficiente. Por otra parte, en la figura 3 se muestra el histograma y superpuesta la función de densidad de probabilidad de los parámetros de la fuerza de avance para un estudio de Monte Carlo de 100 realizaciones donde se observa una distribución normal. Para la implementación de esta rutina se ha utilizado la función fminsearch de la toolbox de optimización de Matlab.

\begin{tabular}{|l|l|l|l|}
\hline & Xudot & $\mathbf{X u}$ & $\mathbf{X u}|\mathbf{u}|$ \\
\hline $\begin{array}{l}\text { Parámetros } \\
\text { Verdaderos }\end{array}$ & -156.1239 & -81.5 & -116.325 \\
$\begin{array}{l}\text { Media } \\
\text { Desviación } \\
\text { estándar. }\end{array}$ & 156.1073 & -81.4893 & -116.3339 \\
& 0.42991 & 0.1115 & 0.0994 \\
\hline
\end{tabular}

Tabla 2 Estudio de Montecarlo de 100 realizaciones distintas. 


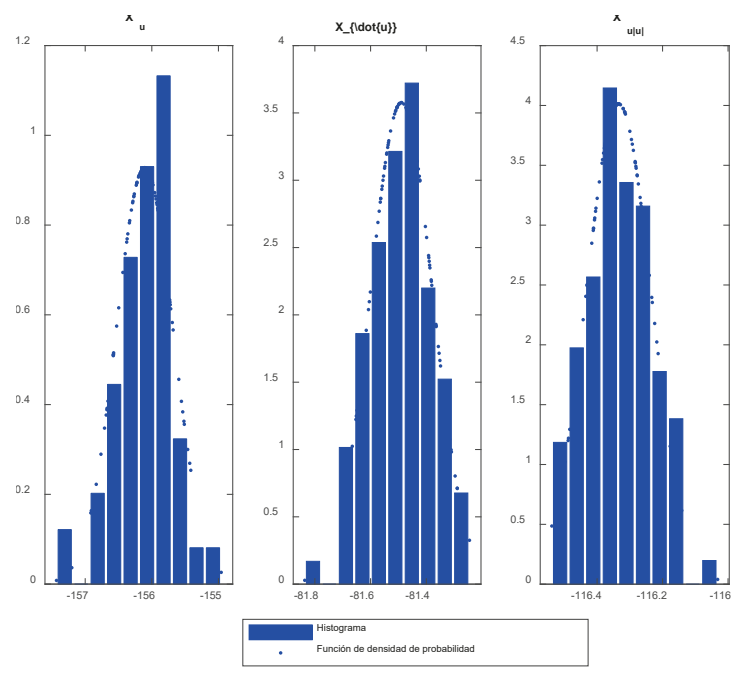

Figura 3: Histograma y función de densidad de probabilidad para un estudio de Monte Carlo de 100 realizaciones.

En el segundo caso se estiman los parámetros utilizando datos adquiridos en los ensayos en canal y se valida el modelo utilizando un conjunto de datos diferente al utilizado en la estimación como es habitual en la aplicación de técnicas de identificación de sistemas. En la figura 4 se muestran los resultados de ajuste a los datos medidos, obtenidos por el método de inferencia indirecta para la estimación de la fuerza de avance.

Otra forma de validar el modelo es mediante el cálculo del coeficiente de determinación, que proporciona información de en qué medida, el modelo que se ha obtenido, es capaz de reproducir los datos medidos, y representa el porcentaje de variaciones de salida reproducidos por el modelo:

$$
R_{2}(\%)=\frac{\sum(\hat{D}-\bar{D})^{2}}{\sum(\hat{D}-D)^{2}+\sum(\hat{D}-\bar{D})^{2}}
$$

Donde D y $\bar{D}$ representan los datos medidos y su media respectivamente, y $\hat{D}$ los datos estimados por el modelo obtenido. Para los datos adquiridos de fuerza de avance se ha obtenido un coeficiente de determinación de $92,3 \%$ que corrobora el buen ajuste mostrado en la figura 4 .

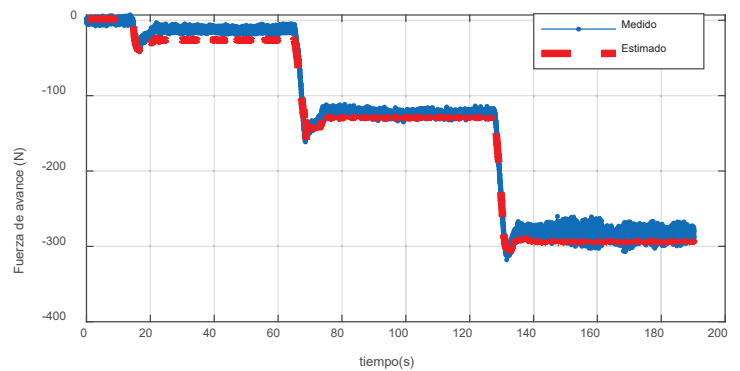

Figura 4: Validación del modelo estimado con datos diferentes a los utilizados en la estimación.

\section{CONCLUSIONES}

En este trabajo se ha propuesto la aplicación de técnicas de inferencia indirecta para la estimación de los coeficientes hidrodinámicos pertenecientes a un modelo de maniobra no lineal de un vehículo subacuático. Se ha realizado un estudio de la aplicación del método para la estimación de la fuerza de desplazamiento vertical del vehículo. Los resultados obtenidos con datos simulados indican valores bajos de sesgo y varianza de los parámetros estimados, inferiores al $1 \%$. Por otro lado, los resultados de validación del modelo con los datos adquiridos en el CEHIPAR muestran un buen ajuste con un valor de coeficiente de determinación cercano al $100 \%$.

\section{Agradecimientos}

Este proyecto ha sido financiado por la Consejería de Universidades, Igualdad, Cultura y Deporte del Gobierno de Cantabria a través del proyecto ControlFond (Control de Vehículos Subacuáticos No Tripulados para Supervisión de Estructuras para Obras Marítimas Fondeadas). (Code: VP45).

\section{English summary}

\section{PARAMETER ESTIMATION OF A NONLINEAR MANEUVERING MODEL OF A REMOTE OPERATED VEHICLE BASED ON BASIN TRIALS}

\begin{abstract}
In this work, indirect inference techniques are applied to estimate the parameters of a nonlinear maneuvering model of a remote operated vehicle (ROV). For this purpose, data from different tests are available, which were carried out with the ROV in the facilities of the Canal de Experiencias Hidrodinámicas del Pardo CEHIPAR, Madrid. The model obtained is validated by means of graphical and statistical methods and the statistical properties of the estimated parameters are evaluated by means of a Monte Carlo study.
\end{abstract}

Keywords: nonlinear maneuvering model; indirect inference; ROV; ordinary least squares.

\section{Referencias}

[1] Allingham, D.; Welsh, J. S.; Godoy, B. I. \& Tusek, J. On the use of indirect inference in equivalent circuit parameter estimation of a synchronous machine 2009 Australasian 
Universities Power Engineering Conference, 2009, 1-5.

[2] Garnier, H. \& Wang, L. (Eds.) Identification of Continuous-Time Models from Sampled Data 1 Springer, 2008, 1-29.

[3] Fossen, T.I. Guidance and control of ocean vehicles. En Libro; Editor, F., USA: Wiley; New York, 1994.

[4] Ghysels E., Khalaf L., and C. Vodounou. Simulation based inference in moving average models. Annales d'Economie et de Statistique, 69:85-100, 2003.

[5] Larsson, C. A.; Hjalmarsson, H. \& Rojas, C. R. Identification of nonlinear systems using misspecified predictors 49th IEEE Conference on Decision and Control (CDC), 2010, 72147219.

[6] Ljung, L., 1987. System identification: theory for the user.

[7] Niyousha Hosseinichimeh \& Hazhir Rahmandad \& Mohammad S. Jalali \& Andrea K. Wittenborn, 2016. "Estimating the parameters of system dynamics models using indirect inference," System Dynamics Review, System Dynamics Society, vol. 32(2), pages 154-178, April.

[8] Yildiz, O. Yilmaz, A. and Gokalp, B. State-ofthe-art system solutions for unmanned underwater vehicles. Radioengineering, 2009, pp.590-600.

[9] Riola, J. La política de I+D en Defensa: Metas y retos tecnológicos. En Las Tecnologías de Doble Uso: La Investigación y el Desarrollo al Servicio de la Sociedad Civil y Militar. España: Centro Universitario de la Defensa (CUD) de San Javier, 2011, pp. 13-22.

[10] Refsnes, J.E.; Sorensen, A.J. Design of control system of torpedo shaped ROV with experimental results. OCEANS '04. MTTS/IEEE TECHNO-OCEAN '04, 2004, pp.264,270.

[11] Revestido E., Velasco F.J., (2012) Two-Step Identification of Nonlinear Manoeuvring Models of Marine Vessels, Ocean Engineering, Volume 53, Pages 72-82.

[12] Revestido Herrero Elías, Velasco Francisco J. and Riola Rodríguez J.M. (2018) Improving parameter estimation efficiency of a non linear manoeuvring model of an underwater vehicle based on model basin data, Applied Ocean Research 76, 125-138, doi: 10.1016/j.apor.2018.04.012.

[13] Revestido Herrero, Elías, Llata García, José Ramón, y Velasco González, Francisco Jesús, Estimación de parámetros de un modelo de maniobra no lineal de un vehículo subacuático no tripulado: un enfoque de inferencia indirecta,
DESEI+D 2018 VI Congreso Nacional De I+D En Defensa Y Seguridad, 2018, Valladolid.

[14] Welsh, J. S.; Agüero, J. C. \& Alamir, M. Continuous-Time System Identification Using Indirect Inference IFAC Proceedings Volumes, $2009,42,1169-1174$.

\section{(c) (1)(9)}

C) 2021 by the authors. Submitted for possible open access publication under the terms and conditions of the Creative Commons Attribution CC BY-NC-SA 4.0 license (https://creativecommons.org/licenses/byncsa/4.0/deed.es). 